\title{
Ketamine-induced cholangiopathy: a case report
}

Wai-Kay Seto ${ }^{1}$, Matthew $\mathrm{Ng}^{2}$, Pierre Chan ${ }^{3}$, Irene Oi-Lin $\mathrm{Ng}^{4}$, Stephen Chi-Wai Cheung $^{5}$, Ivan Fan-Ngai Hung ${ }^{1}$, Man-Fung Yuen ${ }^{1}$, Ching-Lung Lai ${ }^{1}$

${ }^{1}$ Department of Medicine, the University of Hong Kong, Queen Mary Hospital

${ }^{2}$ Department of Medicine, Tung Wah Hospital

${ }^{3}$ Department of Medicine, Ruttonjee Hospital

${ }^{4}$ Department of Pathology, the University of Hong Kong, Queen Mary Hospital

${ }^{5}$ Department of Radiology, Queen Mary Hospital

Correspondence and Reprint request:

Prof. Ching-Lung Lai, Department of Medicine, the University of Hong Kong Queen Mary Hospital, Pokfulam Road, Hong Kong

E mail: hrmelcl@hkucc.hku.hk Fax: 85228162863 Tel: 85222554477 
What is current knowledge:

- Prevalence of recreational ketamine use is increasing.

What is new here:

- Ketamine-induced cholangiopathy with important differential diagnoses excluded. 


\section{To the Editor:}

A 32-year-old lady was referred to our care in April 2006 with on and off epigastric discomfort. She had been inhaling ketamine for recreational use for seven years, and also had lower urinary tract symptoms related to long-term ketamine use. Her liver function was deranged with an elevation of her serum alkaline phosphatase (ALP) and gamma-glutamyl tansferase (GGT) to 229U/L and 284U/L respectively; her bilirubin level and parenchymal liver enzymes were relatively normal. Endoscopic retrograde cholangiopancreatography (ERCP) showed multiple long-segment strictures and narrowing in the intrahepatic ducts of both lobes (Figure 1). The common bile duct was also mildly dilated with no filling defects. The pancreatic duct was normal. ERCP had been repeated nine times over the past 4 years with similar results; brush cytology of dominant strictures obtained each time revealed only reactive changes.

Additional investigations to exclude other causes of biliary disease had been performed. She was negative for anti-mitochondrial antibody (AMA), anti-nuclear antibody (ANA) and anti-HIV antibody. Her serum CA 19.9 and Immunoglobulin G4 (IgG4) levels were normal. A liver biopsy with 12 portal tracts obtained in 2008 showed mild non-specific inflammation of the portal tracts, with no portal or periductal fibrosis (Figure 2). There was no ductopenia to suggest primary biliary cirrhosis (PBC) and no significant portal fibrosis or heavy inflammatory infiltrates to suggest recurrent pyogenic cholangitis (RPC). The classical periductal "onion-skin" fibrosis suggestive of primary sclerosing cholangitis (PSC) was not seen. Colonoscopy with biopsies failed to show any evidence of co-existing inflammatory bowel disease (IBD). 
Ketamine is an N-methyl-D-aspartate (NMDA) antagonist that was first used in clinical practice in the 1970 s as an anaesthetic. Its hallucinogenic side-effects and easy administration by inhalation has made it an increasingly common recreational drug. At low doses, it can result in euphoria and mild sensory and perceptual distortion. Higher doses can result in out-of-body and floating experiences. Its recreational use is extremely common in Asia, especially in Taiwan and Hong Kong. Its prevalence is also on the rise in the United Kingdom (1), especially in the adolescent population.

While long-term ketamine inhalation has been known to be associated with bladder dysfunction (2), its effects on the biliary system have been less well-defined. An animal study has shown ketamine can increase the flow resistance across the sphincter of Oddi (3). There has been only one report of 3 cases with biliary tract abnormalities associated with ketamine use (4), although important differential diagnosis have not been thoroughly excluded in these patients.

We postulate our patient's biliary abnormalities are the result of long-term ketamine usage. Important differential diagnoses for this patient include PBC, PSC, RPC, IgG4-positive sclerosing cholangitis, and AIDS-cholangiopathy. PBC is excluded based on a negative serum AMA and her abnormal cholangiograms. PSC is still a possible diagnosis, although it is a rare disease in Asia and its prevalence outside of Indian and Japan is unknown (5). Moreover, intrahepatic strictures in PSC are usually short and annular in a "beaded" pattern instead of the long strictures present in this patient. There were also no histological features of PSC in her liver biopsy, and coexisting IBD has been excluded. While RPC is a common disease in southeast Asia, the lack of intrahepatic filling defects on multiple cholangiograms and the non-specific 
biopsy findings do not support this diagnosis. A normal serum IgG4 and a negative HIV status have excluded the remaining two differential diagnoses.

In conclusion, long-term ketamine usage can lead to gross biliary abnormalities resulting in recurrent epigastric discomfort and raised ductal enzymes. Clinicians should be aware of this association especially in countries where the recreational use of ketamine is rising.

(600 words)

The authors declare they have participated in the preparation of the case report and have seen and approved the final version.

Conflicts of Interest

All authors have no conflicts of interest

Role of the funding source

All authors have nothing to disclose 


\section{References}

1. McCambridge J, Winstock A, Hunt N, et al. 5-Year trends in use of hallucinogens and other adjunct drugs among UK dance drug users. Eur Addict Res 2007;13:57-64.

2. Tsai TH, Cha TL, Lin CM, et al. Ketamine-associated bladder dysfunction. Int $\mathrm{J}$ Urol 2009;16:826-9.

3. Thune A, Jivegard L, Pollard $\mathrm{H}$, et al. Location of enkephalinase and functional effects of [Leu5]enkephalin and inhibition of enkephalinase in the feline main pancreatic and bile duct sphincters. Clin Sci (Lond) 1992;82:169-73.

4. Wong SW, Lee KF, Wong J, et al. Dilated common bile ducts mimicking choledochal cysts in ketamine abusers. Hong Kong Med J 2009;15:53-6.

5. Ang TL, Fock KM, Ng TM, et al. Clinical profile of primary sclerosing cholangitis in Singapore. J Gastroenterol Hepatol 2002;17:908-13. 